\title{
Transmit / Received Beamforming for Frequency Diverse Array with Symmetrical frequency offsets
}

\author{
Shaddrack Yaw Nusenu* \\ University of Electronic Science and Technology of China, School of Communication and Information Engineering, China. \\ Koforidua Technical University, Electrical / Electronic Department, Faculty of Engineering, Ghana.
}

\begin{tabular}{l} 
A R T I C L E I N F O \\
\hline Article history: \\
Received: 17 February, 2017 \\
Accepted: 07 March, 2017 \\
Online: 15 March, 2017
\end{tabular}

Keywords:

Frequency diverse array

Linear frequency increment

Symmetrical frequency offset

Transmit/received beamforming

\begin{abstract}
A B S T R A C T
Frequency diverse array (FDA) employs a small frequency increment across its array elements to provide range, angle and time dependent beampattern. In most radar systems, FDA with linear frequency offsets have been employed. But, its transmit beampattern is coupled in range and angle dimensions, which may degrade the output signal-tointerference-plus-noise ratio performance. Note that nonlinear offset is also beneficial in FDA radar. In this paper, symmetrical frequency offsets based FDA is investigated which removes the inherent periodicity of FDA beampattern to achieve a single maxima in the target area. In doing so, the signal information at the receiver is improved significantly. Transmit/received beampatterns, signal-to-interference-plus-noise ratio (SINR) and detection probability of the proposed method are compared with the conventional FDA. Computer simulations are used to verify the effectiveness of the proposed method.
\end{abstract}

\section{Introduction}

Frequency diverse array (FDA) utilizing a small frequency increment across each antenna elements was proposed by [1], to steer the beam electronically in angle and range dimensions. The FDA concept has range dependent beamforming capability. It was observed that frequency increment makes the array beampattern changes as a function of the range, angle and time [1]-[3]. In [4], FDA applications for various modes of operations in radar systems has been presented. The periodicity of beam pattern in time, range and angle was investigated in [5]. In [6], a linear FDA was proposed to address the range ambiguous clutters and improves the detection ability in a relatively slow moving targets. The radiation capabilities of FDA was presented in [7] to show its beam scanning features. Also it was proven that the scanning speed was related to frequency offset employed between two neighboring antenna elements. In [8], the range and angle coupled beamforming with frequency diverse chirp signals was presented. In addition, [9] investigated FDA range-angle dependent beamforming to suppress interferences at different ranges and directions.

FDA has a lot of promising application [10] which has sparked many interesting investigations in finding a suitable frequency offset to provide range-angle dependent. It is important to mention

"Shaddrack Yaw Nusenu, UESTC-China and Koforidua Technical University-

Ghana, Email: nusenu2012gh@yahoo.com

www.astesj.com

https://dx.doi.org/10.25046/aj020301 that frequency offset across the FDA elements plays essential role in improving the overall performance of an FDA radar, especially in controlling range-angle dependency and spatial distribution of generated beam pattern [11], [12]. Hence, researchers have shown great interest to investigate frequency offset between the adjacent elements of FDA to improve its performance. Since FDA offers a range-angle dependent beampattern, it is of great significance as this provides a potential for range-angle localization of targets, however, the beampattern of the conventional FDA is coupled in range-angle dimension.

In this regard, a nonuniform linear array was proposed in [13] to decouple the FDA transmit beampattern, however, the carrier frequency and/or frequency increments cannot be altered in realtime because it requires relocating the elements mechanically. In [14] and [15], logarithmically increasing frequency offsets and time-dependent frequency offsets, respectively are reported to decouple the range-angle beampattern. However, these methods resulted in poor beamforming performance in the range dimension. Note that the best decoupling way is to form a dot-shaped beampattern rather than S-shaped beampattern.

In this paper, transmit/received beamforming FDA radar with symmetrical frequency offset to decouple range and angle beampattern in order to produce a single maxima is investigated. The idea is to use the symmetrical frequency offset to provide extra 
degree of freedom in terms of producing single maxima for a particular target of interest in a given region. Due to single maxima the received signal at the receiver array will be better than the signal for the conventional FDA. Furthermore, the proposed transmit/received beamforming are evaluated by the signal-tointerference-plus-noise ratio (SINR) and detection probability. Results shows the superiority of the proposed method.

The remaining sections are organized as follows: Section II presents the signal model of the conventional FDA, followed by the proposed symmetrical frequency offset FDA radar. Section III presents proposed method transmit / received beamforming. Section IV derives the SINR and detection probability. Section V presents results and discussions and concluding summaries are drawn in Section VI.

\section{Background}

\subsection{Conventional FDA Range-Angle Dependent Transmit} Beampattern

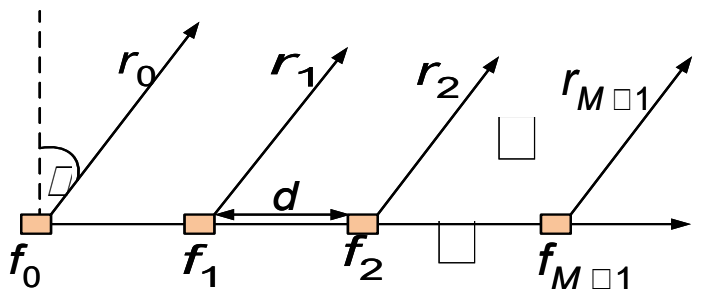

Figure 1: Illustration of linear frequency offset conventional FDA.

Figure 1 shows the conventional FDA antenna array structure. The narrow-band monochromatic signal radiated from each element is identical but with a frequency increment $\Delta f$. The radiated frequency of the $m$ th element can be expressed as

$$
f_{m}=f_{0}+m \mathrm{D} f, \quad m=0,1, \ldots, M-1
$$

where $f_{0}$ is the carrier frequency and $M$ is the number of array elements. Then, the signal transmitted by the mth element can be represented by

$$
s_{m}(\mathrm{t})=\exp \left(-j 2 p f_{m} t\right)
$$

The signal arriving at a far-field point $(r, \theta)$ can be expressed as

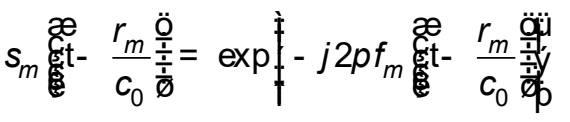

where $c_{0}$ is the speed of light and $r_{m} \| r-m d \sin q$, with $d$ being the element spacing, is the target slant range for the $m$ th element. If uniform weights (all ones) are applied, the array factor can be approximately derived as [16]

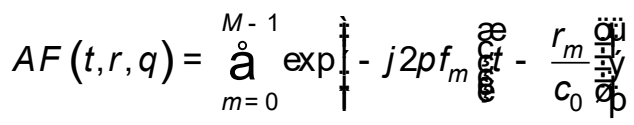

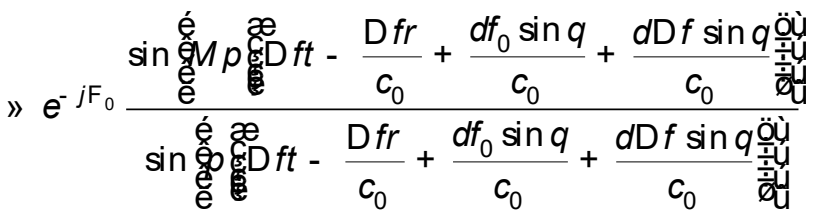

where

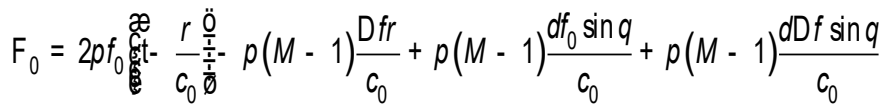

Equation (4) implies that FDA has range, angle and time dependent transmit beampattern. However, the beampattern is coupled in the range and angle dimensions and consequently it will have multiple maximums. To avoid the dependence of range gain on the angle, the FDA parameters should be properly designed.

\subsection{Proposed Symmetrical Frequency offset FDA for Transmit / Received Beamforming}

In this paper, the symmetrical frequency offsets is proposed to decouple range and angle beampattern in order to localize a target in a given region. In designing symmetrical frequency offset, the central element is chosen as the symmetric point depicted in Figure 2.

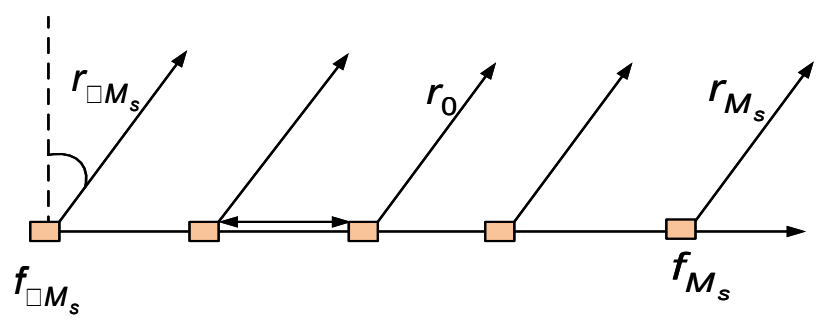

Figure 2: Illustration of proposed symmetrical frequency offset FDA.

In this paper, odd number of elements are assumed, namely, $M=$ $2 M s-1$. The frequency offset for the kth element can be expressed as

$\mathrm{D} f_{k}=|k| \mathrm{D} f, \quad k=-M_{s}+1, \ldots, 0,1, \ldots, M_{s}+1$

Note that in order to show distinct representations, a new element index $k \widehat{\mathrm{I}}\left[-M_{\mathrm{s}}+1, \cdots, 0, \cdots, M_{\mathrm{s}}-1\right]$ instead of $m$ defined in (1) is adopted. Reformulate (4) as

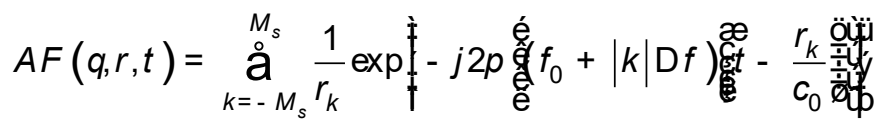

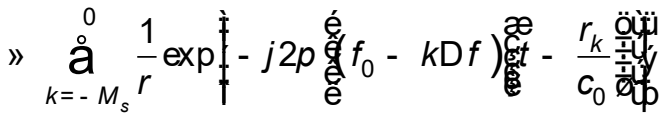

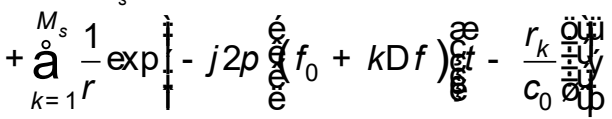


Similarly, $r_{k}=r-k d \sin q$ is used, where $r$ denotes here as the reference range to the central element. Equation (6) can be rewritten in vector notation:

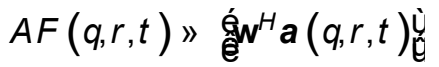

Where ${ }^{H}$ is the conjugate transpose operator, $\mathrm{w}$ is the $M \times 1$ weighting vector, and transmit steering vector a $(\theta, r, t)$ is

$a(q, r, t)=\frac{1}{r} \exp \left(-j F_{-M_{s}}\right), \ldots, 1, \ldots, \exp \left(-j F_{k}\right), \ldots, \exp \left(-j F_{M_{s}}\right) u_{\tilde{U}}^{J}$

with ${ }^{T}$ being the transpose operator and

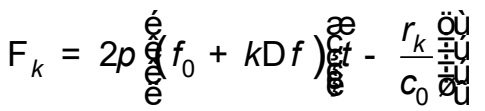

The $\boldsymbol{w}$ can be optimally designed to synthesize the desired transmit beampattern.

\section{Transmit / Received Beamforming for Symmetrical frequency offset FDA}

In this section transmit and received beamforming for proposed method is presented. The transmit beamforming can be expressed as

$$
B_{T}(q, r)=\frac{\left|\mathbf{w}^{H} a(q, r)\right|^{2}}{\left|\mathbf{w}^{H} a\left(q_{d}, r_{d}\right)\right|^{2}}
$$

The normalized received beampattern can be written as

$$
B_{r}(q, r)=\frac{\left|\mathbf{w}_{r}{ }^{H} \mathrm{v}(q, r)\right|^{2}}{\left|\mathbf{w}_{r}{ }^{H} \mathrm{v}\left(q_{d}, r_{d}\right)\right|^{2}}
$$

where $\mathrm{v}(q, r)=\left(\mathbf{w}^{H} \mathrm{a}(q, r)\right) \mathrm{b}(q)$ denotes the virtual steering vector $\mathrm{v}(\theta, r)$ and $\mathrm{b}(\theta)$ being the received steering vector due to the propagation delays from a source to the receive elements.

\section{Performance Analysis}

\subsection{SINR}

The performance analysis of the proposed method are evaluated by signal-to-interference-plus-noise ratio (SINR) and probability of detection. First of all SINR can be expressed as

$$
\operatorname{SINR}=\frac{s_{d}^{2}\left|\mathbf{w}_{r}{ }^{H} \mathrm{v}\left(q_{d}, r_{d}\right)\right|^{2}}{\mathbf{w}_{r}{ }^{H} Q_{i+n} \mathbf{w}_{r}}
$$

where $s_{d}^{2}$ is the variance of the target reflection coefficient and $Q_{i+n}$ denotes the interference-plus-noise covariance matrix which is given as

www.astesj.com

$$
Q_{i+n} @ \stackrel{F}{\stackrel{F}{a}} s_{i}^{2} \mathrm{v}\left(q_{i}, r_{i}\right) \mathrm{v}^{H}\left(q_{i}, r_{i}\right)+s_{n}^{2} I
$$

where $s_{i}^{2}$ is the variance of the $i$ th interference reflection coefficient, $F$ is the number of interferences and $s_{n}^{2} I$ is the covariance matrix with $I$ being an identity matrix. Plugging in (13) and $\mathbf{w}_{r}=\mathrm{v}\left(q_{d}, r_{d}\right)$ into (12) for any radar system, will yields SINR for that radar system.

\subsection{Probability of Detection $(P d)$}

In this section, the probability of detection $(\mathrm{Pd})$ is employed to evaluate the performance of a radar system. For the proposed method, the hypothesis problem can be written as

$\left\{\begin{array}{l}H_{0}: x(t)=n(t) \\ H_{1}: x(t)=h(t)+n(t)\end{array}\right.$

The noise process is assumed to be Gaussian and independent and identically distributed (i.i.d). The probability density function (PDF) can be expressed as

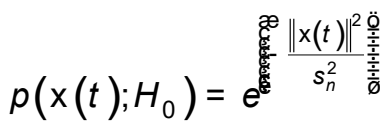

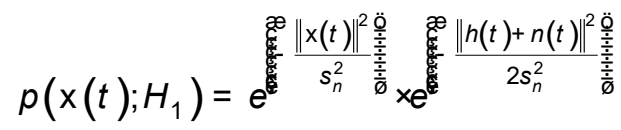

The likelihood ratio test can be given as

$$
g=\frac{p\left(x(t) ; H_{1}\right)}{p\left(x(t) ; H_{0}\right)}<x
$$

The probability of detection $p_{d}$ and probability of false alarm $p_{f a}$, respectively, is expressed as in [17]

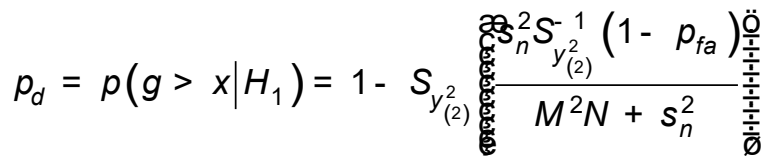

$p_{f a}=p\left(g>x \mid H_{0}\right)=1-S_{y_{(2)}^{2}} \frac{\mathscr{c} 2 x}{2} \frac{\ddot{Q}}{\frac{1}{\dot{\zeta}}}$

where $S(\cdot)$ denotes the cumulative distributive function, $y_{(2)}^{2}$ being the chi-square distribution, $P-N+k$ are the number of elements in $k$ th array and $R$ are the number of receiver array elements. 


\section{Results and Discussions}

In the simulations, the carrier frequency $f_{0}=10 \mathrm{GHz}$, frequency offset $\Delta f=3 \mathrm{KHz}$. Both transmit and receive arrays $M$ and $N$, respectively, have 8 antenna elements spaced by half wavelength $d=\frac{c_{0}}{2 f_{0}}$. The target is assumed to be located at $\left(\theta_{d}, r_{d}\right)=\left(50^{\circ}, 50\right.$

$\mathrm{km})$. Gaussian noise is utilized as additive noise with zero mean spatially and temporally white random sequence having same variance on each array elements.

\section{A. Transmit Beampattern}

First, normalized transmit beampattern for FDA and the proposed symmetrical FDA is presented to show the effectiveness of symmetrical FDA. Note that the two arrays are steered to instantaneous angle $\theta=0^{\circ}$ and the beam direction ranges are also normalized to zero-range. Figure 3(a) illustrates the conventional FDA beampattern which is coupled in the range-angle dimension.

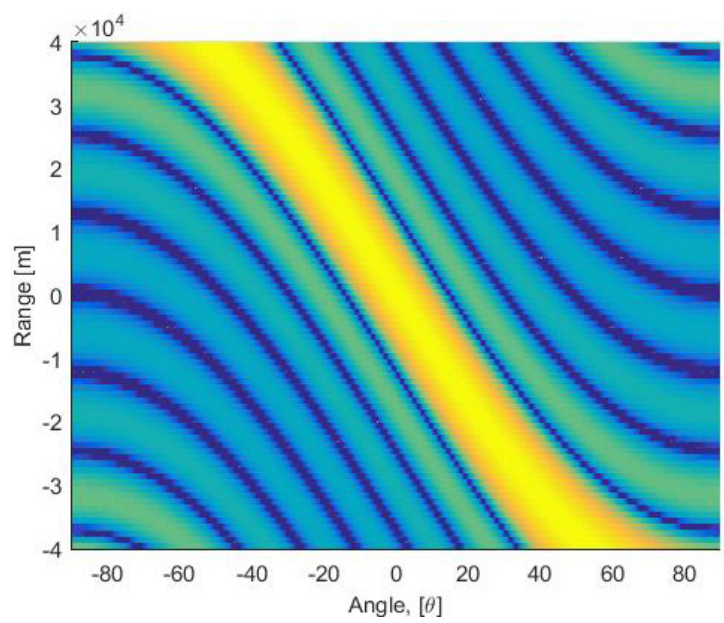

(a)

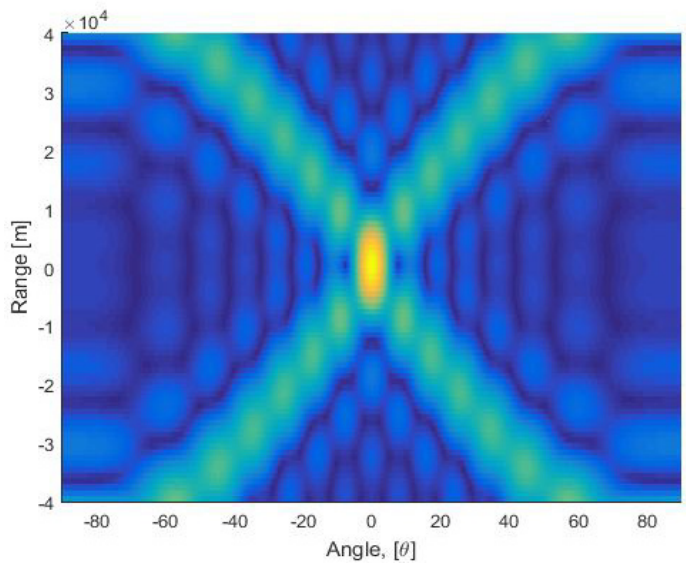

(b)

Figure 3: Comparisons of normalised transmit beampattern when $\Delta f=3 \mathrm{KHz}$ is adopted: (a) Conventional FDA, (b) Proposed symmetrical FDA.

In contrast, Figure 3(b) shows the proposed symmetrical FDA which has decoupled range-angle beampattern. And this is beneficial for targets localization.

As shown in Figure 4(a), the conventional FDA beampattern shows the problem of periodic maxima. This maxima are www.astesi.com undesirable for proper target localization. On the other hand, Figure 4(b) shows the proposed method beampattern which produce exactly one maxima at target location and no other maxima is seen in the entire region of observation.

\section{B. Received Beampattern}

In Figure 5(a) and 5(b) both radars produce maxima at the intended range-angle pair, but the conventional FDA has more serious ambiguity problem shown by the arrow in Figure 5(a). It is important to mention that because the conventional FDA has larger total frequency offset, it achieved higher range resolution than the proposed method. In the case of the proposed method, the target is better localized than the conventional FDA. It can be noticed that there is no spread of beampattern in both range and angle dimensions.

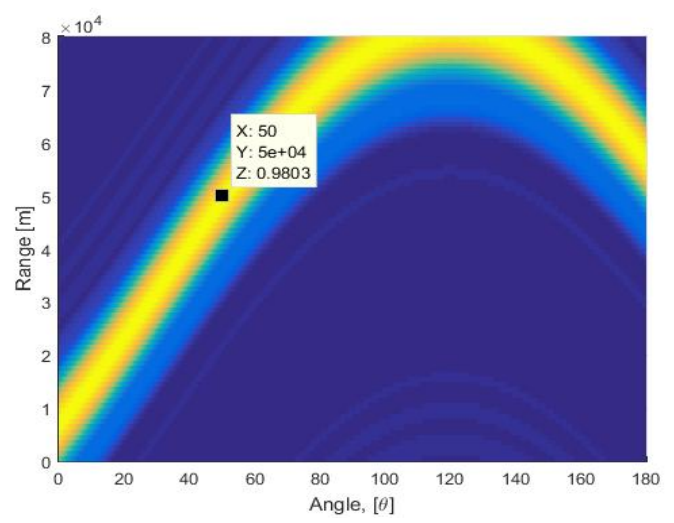

(a)

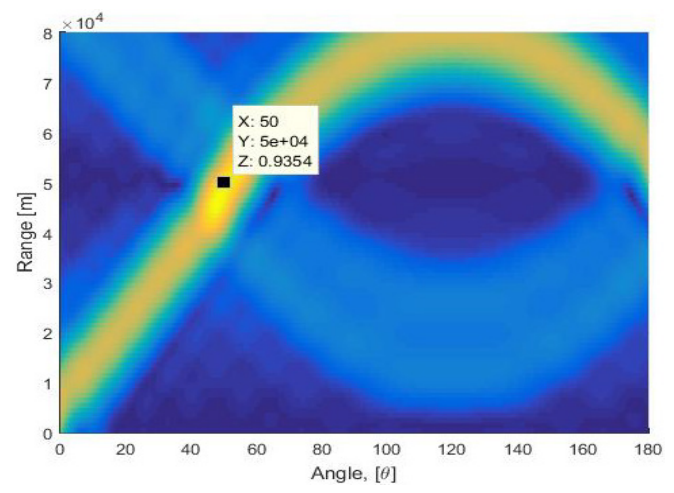

(b)

Figure 4: Comparisons of transmit beampattern when the target is present at $\left(\theta_{d}, r_{d}\right)$ $=\left(50^{\circ}, 50 \mathrm{~km}\right)$ : (a) Conventional FDA, (b) Proposed symmetrical FDA.

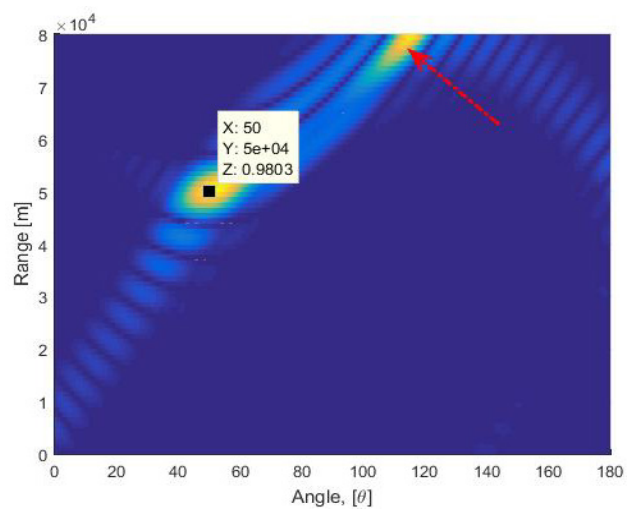

(a) 


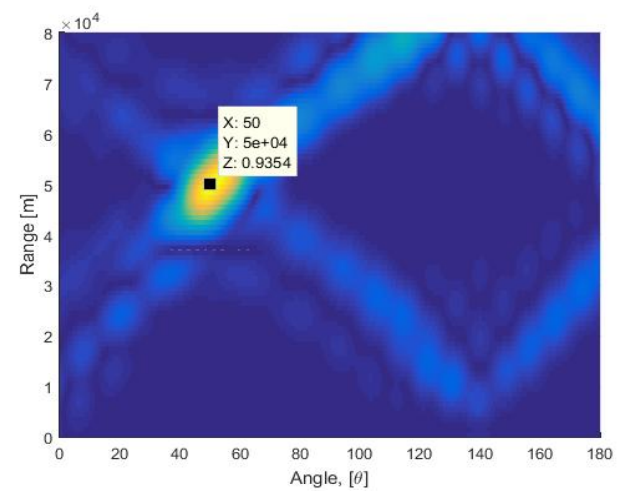

(b)

Figure 5: Comparisons of transmit beampattern when the target is present at $\left(\theta_{d}, r_{d}\right)$ $=\left(50^{\circ}, 50 \mathrm{~km}\right)$ : (a) Conventional FDA, (b) Proposed symmetrical FDA.

Finally, SINR and probability detection performance of the proposed method and the conventional FDA have been plotted. It can be observed that in Figure 6, the SINR of proposed symmetrical FDA is better than the conventional FDA. Thus proposed method has better robustness against the interferences.

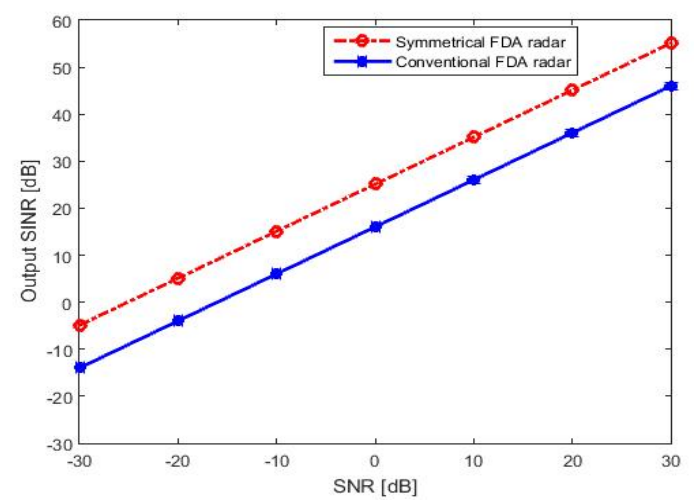

Figure 6: SINR versus SNR performance.

Figure 7 shows the detection probability versus SNR for the proposed method and the conventional FDA radar. Proposed method exhibits better detection performance compared to the conventional FDA radars.

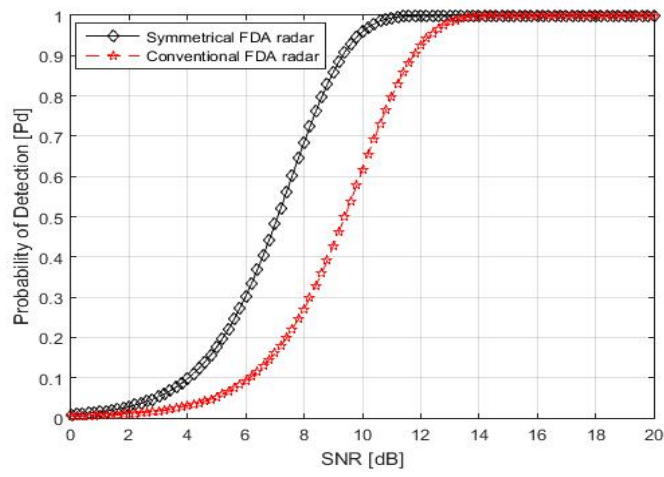

Figure 7: Probability of detection versus SNR performance.

Note that the improvement in performance in terms of SINR and detection probability can be attributed to single maxima due to symmetrical frequency offset employed across the transmit antenna array.

\section{Conclusion}

A symmetrical frequency offset based FDA has been presented. The proposed method avoids the periodic maxima presented in conventional FDA radar and focus the target with multiple beams to get single maxima for better target localization. Numerical results shows that the proposed method exhibits better robustness against interference as well as better detection performance than the conventional FDA radar due to the employment of symmetrical frequency offset across the transmit antenna array. Overall, the proposed method received beampattern show a reasonable improvement compared to the conventional FDA.

\section{Conflict of Interest}

The author declare no conflict of interest.

\section{Acknowledgment}

The author would like to thank Respected Professor Wen-Qin Wang (Senior Member - IEEE) of UESTC for his great contributions, support and encouragement.

\section{References}

[1] Antonik, P., Wicks, M.C., Griffiths, H.D., et al. "Frequency diverse array radars," Proc. of the IEEE Radar Conf., Verona, NY, April 2006, pp. 215-217

[2] Wicks, M.C., Antonik, P. "Frequency diverse array with independent modulation of frequency, amplitude, and phase," U.S.A Patent 7,319,427, 15 January 2008

[3] Wicks, M.C., Antonik, P. "Method and apparatus for a frequency diverse array," U.S.A Patent 7.511, 665B2, 31 March 2009

[4] Antonik, P., M. C. Wicks, H. D. Griffiths, and C. J. Baker. "Multi-mission multimode waveform diversity," Proc. IEEE Radar Conf. Dig., 580582, Verona, NY, USA, Apr. 2006, pp. 24-27.

[5] Secmen M., Demir S., Hizal A., Eker T. "Frequency diverse array antenna with periodic time modulated pattern in range and angle," IEEE Conference on Radar, 2007

[6] Wen-Qin Wang. "Range-Angle Dependent Transmit Beampattern Synthesis for Linear Frequency Diverse Arrays," IEEE Transactions Signal Processing. 61 (8), 2013, pp. 4073-4081.

[7] Huang S., Tong K. F., Baker C. J. "Frequency diverse array with beam scanning feature,” Proc. IEEE Antennas Propag. Conf., USA, July 2008.

[8] Higgins T. and S. Blunt. "Analysis of range-angle coupled beamforming with frequency diverse chirps," Proceedings of the 4th International Waveform Diversity and Design Conference, Orlando, FL, Feb. 2009, pp. 140-144.

[9] Huaizong Shao, Jingchi Li, Hui Chen, and Wen-Qin Wang. "Adaptive Frequency Offset Selection in Frequency Diverse Array Radar," IEEE Antennas and Wireless Propagation Letters, vol. 13, 2014.

[10] W.-Q. Wang. "Overview of frequency diverse array in radar and navigation applications," IET Radar, Sonar and Navigation, DOI: 10.1049/ietrsn.2015.0464, vol. 10, 2016.

[11] Zhuang, L. and X. Z. Liu. "Precisely beam steering for frequency diverse arrays based on frequency offset selection,” Proc. Int. Radar Conf., pp. 1-4, 2009.

[12] Chen, Y.-G., Y.-T. Li, Y.-H. Wu, and H. Chen. "Research on the linear frequency diverse array performance," Proc. IEEE 10th Int. Conf. on Signal Processing, 23242327, Beijing, Oct. pp. 24-28, 2010.

[13] W.-Q. Wang, H. C. So, and H. Z. Shao. "Nonuniform frequency diverse array for range-angle imaging of targets," IEEE Sensors Journal, vol. 14, no. 8, pp. 24692476, August 2014. 
[14] W. Khan, I. M. Qureshi, and S. Saeed. "Frequency diverse array radar with logarithmically increasing frequency offset," IEEE Antennas and Wireless Propagation Letters, vol. 14, 2015

[15] W. Khan and I. M. Qureshi. "Frequency diverse array radar with time dependent frequency offset," IEEE Antennas and Wireless Propagation Letters, vol. $13,2014$.

[16] Sammartino, P.F., Baker, C.J., Griffiths, H.D. "Frequency diverse MIMO techniques for radar," IEEE Trans. Aerosp. Electron. Syst., 2013, 49, (1), pp. 201222.

[17] W. Q. Wang and H. Shao. "Range-angle localization of targets by a doublepulse frequency diverse array radar," Sel. Top. Signal Process IEEE J., vol. 8, no. 1, pp. 106-114, 2014. 